\title{
Investigation of dynamics of self-similarly evolving magnetic clouds
}

\author{
G. Dalakishvili1 ${ }^{1,4}$, A. Rogava ${ }^{2,3}$, G. Lapenta ${ }^{4}$, and S. Poedts ${ }^{4}$ \\ ${ }^{1}$ Institut für Weltraum und Astrophysik, Ruhr-Universität Bochum (RUB), Germany \\ e-mail: giorgi@tp4.rub.de \\ 2 Centre for Theoretical Astrophysics, ITP, Ilia State University, Georgia \\ e-mail: andria.rogava@iliauni.edu.ge \\ 3 Abdus Salam International Centre for Theoretical Physics, Italy \\ 4 Centre for Plasma Astrophyics, Katholieke Universiteit Leuven, Belgium \\ e-mail: [Giovanni.Lapenta; Stefaan.Poedts]@wis.kuleuven.be \\ Received 20 April 2010 / Accepted 9 October 2010
}

\section{ABSTRACT}

\begin{abstract}
Context. Magnetic clouds (MCs) are "magnetized plasma clouds" moving in the solar wind. MCs transport magnetic flux and helicity away from the Sun. These structures are not stationary but experience temporal evolution. Simplified MC models are usually considered.

Aims. We investigate the dynamics of more general, radially expanding MCs. They are considered as cylindrically symmetric magnetic structures with low plasma $\beta$.

Methods. We adopt both a self-similar approach method and a numerical approach.

Results. We demonstrate that the forces are balanced in the considered self-similarly evolving, cylindrically symmetric magnetic structures. Explicit analytical expressions for magnetic field, plasma velocity, density, and pressure within MCs are derived. These solutions are characterized by conserved values of magnetic flux and helicity. We also investigate the dynamics of self-similarly evolving MCs by means of the numerical code "Graale". In addition, their expansion in a medium of higher density and higher plasma $\beta$ is studied. It is shown that the physical parameters of the MCs maintain their self-similar character throughout their evolution.

Conclusions. After comparing different self-similar and numerical solutions, we are able to conclude that the evolving MCs are quite adequately described by our self-similar solutions - they retain their self-similar, coherent nature for quite a long time and over large distances from the Sun.
\end{abstract}

Key words. magnetohydrodynamics (MHD) - magnetic fields - plasmas - solar wind

\section{Introduction}

It is well-known that coronal mass ejections (CMEs) are one of the most significant forms of solar activity. They carry enormous masses of plasma threaded by the magnetic field away into the interplanetary medium. Further away from the Sun, these large-scale, dynamical plasma structures are commonly called interplanetary coronal mass ejections (ICMEs). Magnetic clouds (MCs) form a subset of ICMEs (Klein \& Burlaga 1982; Burlaga 1991; Farrugia et al. 1995). Spacecrafts crossing the central parts of these MCs provide valuable information about their physical characteristics. It turns out that MCs have a strong magnetic field, low proton temperatures (low plasma $\beta$, compared to the ambient solar wind with the same speed) and they exhibit a substantial and smooth rotation of the magnetic field vector. These three features of MCs are selected as signatures of MCs (Nakwacki et al. 2008). The MCs are also characterized by a coherence of the magnetic field (low level of fluctuations). The radial dimension of a $\mathrm{MC}$ is typically $\approx 0.25 \mathrm{AU}$ (at $1 \mathrm{AU}$ ).

These in situ observations of the physical properties of MCs are considered important steps towards the prediction of the geophysical effectiveness of their interaction with the Earth's magnetosphere, for space weather forecasts and related issues.

Different models for the structures of magnetic clouds have been proposed. There is no general agreement about the large-scale structure of MCs. The local structure of MCs are often assumed to follow of cylindrically symmetric force-free configurations (Burlaga 1988, 1991; Demoulin \& Dasso 2009). It is often suggested that the ends of MCs connect to the surface of the Sun, while, according to other models, MCs are described as tori (Vandas et al. 2006, 2009; Romashets et al. 2006, 2007). In a number of studies, MCs are assumed to be force-free, static, axially symmetric flux ropes and their magnetic field is constructed on the basis of Lundquist's model (Burlaga 1988; Lepping et al. 1990; Farrugia et al. 1993). Observations show, however, that MCs do not stay static but expand while propagating in the solar wind and they continue to expand well beyond 1 AU (Burlaga 1991; Demoulin 2008; Demoulin \& Dasso 2009; Bothmer \& Schwenn 1998). In a vast majority of the cases it is observed that the frontal parts of the MCs propagate at larger velocities than their back regions. This illustrates that, with respect to the MC's own cylindrical set of coordinates, the radial size of those cylindrical MCs increases (Nakwacki et al. 2008). Theoretical models including the effect of radial expansion have been proposed before (Osherovich et al. 1995; Farrugia et al. 1993; Nakwacki et al. 2008). In these models, only the radial expansion is taken into account and solutions have been found for all plasma parameters. There are other studies (Shimazu \& Vandas 2002; Demoulin \& Dasso 2009), however, where the axial expansion is also included. 
Previous studies demonstrated that inside MCs the density drops as $d^{-2.4}$ (Bothmer \& Schwenn 1998), i.e., the volume of MC increases as $d^{2.4}$, where $d$ denotes the distance from the Sun. The radius of the MCs, denoted by $(R)$, was found to increased and at a rate that varied with the distance, viz. as: $R \sim d^{0.8}$ (Bothmer \& Schwenn 1998). Since the surface of the MC's cross-section perpendicular to its axis increases as $R^{2} \sim d^{1.6}$, and the MC's volume increases as $d^{2.4}$, the MC's longitudinal size should increase as $d^{0.8}$. Therefore, according to Bothmer \& Schwenn's data, the MCs are radially expanding and also show an extension along their axis.

In the present study, we consider self-similarly expanding cylindrical MCs that are able to expand in both the radial and longitudinal directions. We consider the problem in the frame of the $\mathrm{MC}$ and in cylindrical coordinates related to the MC, i.e. with a longitudinal axis $Z$ that coincides with the MC's axis. Overall cylindrical symmetry of the MC is assumed. Based on these assumptions, we derive the appropriate full set of non-stationary MHD equations and find their analytical solutions. The logical and natural consequence of the assumptions of self-similarity and cylindrical symmetry is that the dynamic forces acting upon the MCs are balanced. The solutions include expressions for the plasma magnetic field, velocity, mass density, and thermal pressure.

An important feature of our model is that certain significant characteristics of the MCs - magnetic flux and helicity - are conserved. We also separately consider the particular case of a $\mathrm{MC}$ that is allowed to expand only in the radial direction. It can be shown that in this case, the MHD equations do not have any physical, self-similar solution.

\section{Self-similar expanding MC models}

\subsection{General equations and self-similar expansion}

To perform an analytic study of the dynamics of magnetic clouds, we have to start from the full set of MHD equations

$\nabla \cdot \boldsymbol{B}=0$,

$\partial_{t} \boldsymbol{B}=\nabla \times[\boldsymbol{V} \times \boldsymbol{B}]$,

$\partial_{t} \varrho+\nabla \cdot(\varrho \boldsymbol{V})=0$

$\varrho\left[\partial_{t}+(\boldsymbol{V} \cdot \nabla)\right] \boldsymbol{V}=(1 / 4 \pi)(\nabla \times \boldsymbol{B}) \times \boldsymbol{B}-\nabla \cdot p$.

In these equations, $p$ denotes the thermal plasma pressure, $\varrho$ is the density, $\boldsymbol{V}$ is the velocity field, and $\boldsymbol{B}$ denotes the magnetic field.

In a number of previous studies, the MCs were considered as cylindrical magnetic structures, characterized by axial symmetry. In the present consideration, both symmetry along the $Z$ axis $\left(\partial_{z}=0\right)$ and the azimuthal symmetry $\left(\partial_{\varphi}=0\right)$ are assumed. The axially symmetric magnetic field can then be expressed as

$\boldsymbol{B} \equiv\left[\begin{array}{lll}0, & B_{\varphi}, & B_{z}\end{array}\right]$,

where $B_{\varphi}=B_{\varphi}(r, t)$ and $B_{z}=B_{z}(r, t)$. We note that this representation satisfies the solenoidal condition.

The self-similar approach, adopted here, implies that the temporal evolution of the physical functions is controlled by the self-similar variable

$\xi=\frac{r}{\Phi(t)}$, where $\Phi(t)$ denotes a function of time. Let us search solutions of the MHD equations in the following form (in analogy with Low 1982):

$B_{\varphi}=\Phi^{\delta} Q_{\varphi}(\xi)$,

$B_{z}=\Phi^{\sigma} Q_{z}(\xi)$,

$\varrho=\Phi^{\alpha} \tilde{\rho}(\xi)$,

$p=\Phi^{\beta} \tilde{p}(\xi)$.

One can see that, the type of solutions introduced by Eqs. (7a)(7d) evolve self-similarly and are characterized by a particular time-scaling. Here $Q_{\varphi}, Q_{z}, \tilde{\rho}$, and $\tilde{p}$ are functions of the selfsimilar variable $\xi$, and $\Phi^{\delta}, \Phi^{\sigma}, \Phi^{\alpha}$, and $\Phi^{\beta}$ show the time scaling of the azimuthal and longitudinal components of the magnetic field, the plasma density, and the plasma pressure, respectively.

\subsection{Solution of the induction equation}

We consider both a radial and a longitudinal expansion of the $\mathrm{MC}$ but no motion in the azimuthal direction. In this case, the Eulerian velocity field of the plasma, $\boldsymbol{V}$, can be expressed as

$\boldsymbol{V}=\left[\begin{array}{lll}V_{r}, & 0, V_{z}\end{array}\right]$,

where, we assume that the radial component of the velocity $V_{r}=$ $V_{r}(r, t)$, and the $z$-component $V_{z}=V_{z}(z, t)$, i.e. we assume that the MC maintains its cylindrical shape during its evolution.

After substitution of Eq. (8) in Eq. (2), we derive

$\partial_{t} B_{z}+\frac{1}{r} \partial_{r}\left(r V_{r} B_{z}\right)=0$.

After taking into account Eqs. (6), (7b) and the Eqs. (A.1) and (A.2) given in the appendix, Eq. (9a) can be rewritten as

$Q_{z}\left[\sigma \dot{\Phi}+\frac{V_{r}}{\xi}+\Phi \partial_{r} V_{r}\right]+Q_{z}^{\prime}\left[V_{r}-\xi \dot{\Phi}\right]=0$,

where $Q_{z}^{\prime}$ corresponds to $\mathrm{d} Q_{z}(\xi) / \mathrm{d} \xi$. Equation (9a) (hence Eq. (9b)) is satisfied for arbitrary $Q_{z}$ only when

$V_{r}-\xi \dot{\Phi}=0$,

and

$\sigma \dot{\Phi}+\frac{V_{r}}{\xi}+\Phi \partial_{r} V_{r}=0$

From Eqs. (10a) and (10b), we can infer that the radial component of the Eulerian plasma velocity is described as

$V_{r}=r \dot{\Phi} / \Phi$

and

$\sigma=-2$

where $\Phi$ is the function of time mentioned in Eq. (6).

One can check that for $\sigma=-2$ the longitudinal magnetic flux $\phi_{z}$ is conserved. Nakwacki et al. (2008) analyzed different MC models and derived expressions for the magnetic flux, the magnetic helicity, and the magnetic energy per unit length along the flux tube. The models that are in good agreement with observations are characterized by the conservation of $\phi_{z}$ (see also Berdichevsky et al. 2003).

We now analyze the $\varphi$-component of the induction equation, Eq. (2)

$\partial_{t}\left(B_{\varphi}\right)+B_{\varphi} \partial_{z} V_{z}+\partial_{r}\left(V_{r} B_{\varphi}\right)=0$. 
The combination of Eqs. (11) and (13) leads to the important relation

$(\delta+1) \dot{\Phi} / \Phi+\partial_{z} V_{z}=0$.

After taking into account Eqs. (5) and (8) in addition with the assumption of azimuthal symmetry, one can see that the radial component of the induction equation, Eq. (2), is automatically satisfied and does not lead to any additional restrictions.

\subsection{Self-similar solutions}

After inserting the expressions for the plasma density given by Eq. (7c), the velocity introduced by Eq. (8), and the radial component of velocity defined in Eq. (11), into the mass conservation law given by Eq. (3), we obtain another important relation, viz.

$(\alpha+2) \dot{\Phi} / \Phi+\partial_{z} V_{z}=0$.

To ensure consistency between Eqs. (14) and (15), one should obviously have $\alpha+2=\delta+1$.

The $z$-component of the equation of motion, Eq. (4), helps us to derive an expression for the $z$-component of the plasma velocity

$\partial_{t} V_{z}+V_{z} \partial_{z} V_{z}=0$

We try to solve the partial differential equation in Eq. (16a) by using the variable separation technique, i.e. we assume that

$V_{z}(z, t)=Z(z) T(t)$.

Substitution of Eq. (16b) in Eq. (16a) yields:

$\dot{T} Z+Z T^{2} Z^{\prime}=0$,

where $\dot{F} \equiv \partial_{t} F$ denotes the first-order time derivative of a function $F$. Hereafter we use, to indicate second order derivatives, the notation $\ddot{F} \equiv \partial_{t}^{2} F$, Where $Z^{\prime}$ presents $\mathrm{d} Z / \mathrm{d} z$.

It follows from Eq. (17a) that

$-\frac{\dot{T}}{T^{2}}=Z^{\prime}=$ const.

Equation (17b) can be decomposed into two ODEs, viz.

$-\frac{\dot{T}}{T^{2}}=\lambda$

and

$Z^{\prime}=\lambda$,

where, $\lambda$ is an arbitrary constant.

After solving the ODEs (17c)-(17d) with the assumption that, at the surface $z=0, V_{z}=0$, we derive the expressions

$T=\frac{T_{0}}{1+\lambda T_{0} t}$

and

$Z=\lambda z$

where $\lambda$ and $T_{0}$ are constants.

After inserting Eqs. (18a)-(18b) into Eq. (16b), we obtain our desired expression for $V_{z}$ :

$V_{z}=\frac{z k}{1+k t}$, where, $k \equiv \lambda T_{0}$.

We assumed that locally the MC could be described as a cylindrical structure. We investigate the evolution of the length $L$ of this cylindrical structure. For this purpose, we describe the temporal evolution of the $z$-coordinate of the plasma element located at the position $z=L$ at time $t$. The Lagrangian velocity of this element coincides with the Eulerian velocity of the plasma flow at time $t$ and $z=L$. If at a certain time the coordinate of this element is $L$, then its Lagrangian velocity is

$V_{L}=\frac{\mathrm{d} L}{\mathrm{~d} t}$.

From Eq. (19), we then have that

$\frac{\mathrm{d} L}{\mathrm{~d} t}=\frac{L k}{1+k t}$.

The solution of this ordinary differential equation in Eq. (20b) gives an expression for the longitudinal size of the considered cylindrical structure of

$L=L_{0}(1+k t)$

where $L_{0}$ is the length of the cylinder at $t=0$.

The radial component of the equation of motion, in combination with the expressions for the magnetic field, the velocity, and the plasma density lead to

$\Phi^{\alpha} \xi \ddot{\Phi} \tilde{\rho}=F_{r}$,

where, $F_{r}$ denotes the radial component of the total force. In terms of $\tilde{p}, Q_{\varphi, z}$, and $\xi$, this force component can be expressed as (details of the derivation are given in the appendix)

$F_{r}=-\frac{1}{4 \pi}\left[\frac{1}{\Phi^{5}} Q_{z}^{\prime} Q_{z}+\Phi^{\delta-3}\left(\frac{Q_{\varphi}^{2}}{\xi}+Q_{\varphi}^{\prime} Q_{\varphi}\right)\right]-\Phi^{\beta-1} \tilde{p}^{\prime}$,

where $Q_{\varphi, z}^{\prime}=\mathrm{d} Q_{\varphi, z} / \mathrm{d} \xi$ and $\tilde{p}^{\prime}=\mathrm{d} \tilde{p} / \mathrm{d} \xi$. To self-consistent time scaling for all terms in Eq. (22), one has to assume that $\delta=-2$ and $\beta=-4$. By a comparising of Eqs. (14) and (15), we find that if $\delta=-2$, then $\alpha=-3$.

From Eqs. (15) and (19), we find that

$\frac{\dot{\Phi}}{\Phi}=\frac{k}{1+k t}$.

Equation (23) is an ordinary differential equation (ODE) in terms of $\Phi(t)$. After solving this ODE, we find the explicit expression for $\Phi(t)$

$\Phi=\Phi_{0}(1+k t)$,

where $\Phi_{0}$ is a constant parameter.

The substitution of expression Eq. (24) into Eq. (21) leads to an important conclusion: calculating the magnetic and pressure gradient forces, we see that for the self-similarly evolving, cylindrical, axially-symmetric structure, the magnetic force $F_{\mathrm{m}} \equiv 1 /(4 \pi)(\nabla \times \boldsymbol{B}) \times \boldsymbol{B}$ and the thermal pressure gradient force $F_{\mathrm{p}} \equiv-\nabla \cdot p$ are exactly balanced, i.e.,

$F_{r}=F_{\mathrm{m}}+F_{\mathrm{p}}=0$.

If we associate the value $\xi_{0}$ of the self-similar variable $\xi$ with the boundary of the MC, then the expression of the MC Lagrangian velocity is given by (Low 1982):

$V_{s}=\frac{\mathrm{d} R}{\mathrm{~d} t}=\xi_{0} \frac{\mathrm{d} \Phi}{\mathrm{d} t}$. 
After substituting Eq. (24) for $\Phi$ into Eq. (26), we can derive a time-dependent solution for the $\mathrm{MC}$ radius:

$R=R_{0}(1+k t)$.

We note that the form of this expression coincides with the one given by Nakwacki et al. (2008).

\subsection{Plasma and force-free field evolution}

The remaining solutions readily follow from the derived equations, yielding

$$
\begin{aligned}
V_{r} & =\frac{r k}{1+k t}, \\
\varrho & =\frac{\tilde{\varrho}}{(1+k t)^{3}},
\end{aligned}
$$

and

$$
p=\frac{\tilde{p}}{(1+k t)^{4}}
$$

where, $\tilde{\varrho}$ and $\tilde{p}$ are arbitrary functions of $\xi=r / \Phi$.

After analyzing of the expressions for pressure and density given by Eq. (28b), (28c), one can check that, for systems characterized by entropy conservation, the entropy conservation law is satisfied only if the polytropic index $\gamma=4 / 3$. This is indeed a common feature of all different self-similar systems (Low 1982; Farrugia et al. 1995; Finn et al. 2004).

From various observations, it is known that MCs are characterized by low plasma $\beta$ 's (Burlaga et al. 1981; Burlaga 1991; Bothmer \& Schwenn 1998). The thermal pressure term in the total force could be neglected and this implies that we have to construct a force-free magnetic field that evolves in a selfsimilar way. The cylindrically symmetric force-free structure of the MC's magnetic field is indeed advocated by a number of studies (Burlaga 1988; Lepping et al. 1990; Farrugia et al. 1993; Farrugia et al. 1995; Nakwacki et al. 2008; Demoulin \& Dasso 2009). A force-free magnetic field complies with the relation

$$
\nabla \times \boldsymbol{B}=\mu \boldsymbol{B} .
$$

If we rewrite the vectorial equation in Eq. (29) for each component of vectors, taking in to account Eqs. (7a), (7b), and (12), we obtain

$-Q_{z}^{\prime}=\mu \Phi Q_{\varphi}$,

$Q_{\varphi}^{\prime}+\frac{Q_{\varphi}}{\xi}=\mu \Phi Q_{z}$

where $Q_{\varphi, z}^{\prime}$ stands for $\mathrm{d} Q_{\varphi, z} / \mathrm{d} \xi$. If we take the derivative of both terms of Eq. (30a) with respect to the variable $\xi$, we get

$-Q_{z}^{\prime \prime}=\mu \Phi Q_{\varphi}^{\prime}$,

where it is assumed that $\mu$ does not depend on $\xi$. In general, however, $\mu$ could be a function of $\xi$.

If we take in to account Eqs. (30a) and (30c), we can derive from Eq. (30b) an ordinary differential equation for $Q_{z}$

$Q_{z}^{\prime \prime}+\frac{Q_{z}^{\prime}}{\xi}+\mu^{2} \Phi^{2} Q_{z}=0$

With the following transformation of variables $x=\mu \Phi \xi$, we can rewrite Eq. (31a) as

$\frac{\mathrm{d}^{2} Q_{z}}{\mathrm{~d} x^{2}}+\frac{1}{x} \frac{\mathrm{d} Q_{z}}{\mathrm{~d} x}+Q_{z}=0$.
Actually Eq. (31b) being a Bessel equation of zero order, with the solution

$Q_{z}=J_{0}(x)=C_{0} J_{0}(\mu \Phi \xi)$,

where $J_{0}(x)$ is the Bessel function of the first kind and $C_{0}$ is a constant parameter. We note that the solution that is not characterized with a singularity at $x=0$ has been chosen. The substitution of Eq. (32a) into Eq. (30a) leads to an expression for $Q_{\varphi}$

$Q_{\varphi}=J_{1}(x)=C_{0} J_{1}(\mu \Phi \xi)$,

where $J_{1}(x)$ is the Bessel function of the first kind.

From Eqs. (32a), (32b), we see that $Q_{\varphi}$ and $Q_{z}$ are functions of $\mu \Phi \xi$. Since we assumed above that $\mu$ is not a function of $\xi$, that $\Phi$ is only a function of $t$ (Eq. (6)), and that $Q_{\varphi, z}$ are functions only of $\xi$ (Eqs. (7a), (7b)), it follows that $\mu \Phi=$ const. The substitution of Eqs. (32a), (32b), (24), and (6) into Eqs. (7a) and (7b), respectively, taking into account that $\delta=\sigma=-2$, leads to the following expressions for the components of the magnetic field

$B_{r}=0$,

$B_{\varphi}=\frac{B_{0}}{(1+k t)^{2}} J_{1}\left(\frac{r}{r_{0}(1+k t)}\right)$,

and

$B_{z}=\frac{B_{0}}{(1+k t)^{2}} J_{0}\left(\frac{r}{r_{0}(1+k t)}\right)$,

where $B_{0}$ and $r_{0}$ are constants, and $C_{0} / \Phi_{0}^{2}$ has been substituted by $B_{0}$ and $\mu \xi / \Phi_{0}$ is substituted by $1 / r_{0}$.)

From Eqs. (33a)-(33c), we can calculate important expressions for the magnetic flux and the helicity (Nakwaci et al. 2008) associated with the MC

$\Phi_{z}=\frac{2 \pi}{\chi} R \frac{B_{0}}{(1+k t)^{2}} J_{1}(\chi R)$,

$\Phi_{\varphi}=\frac{1}{\chi} \frac{B_{0}}{(1+k t)^{2}} L\left(1-J_{0}(\chi R)\right)$,

and

$H=\frac{2 \pi}{\chi} R^{2} \frac{B_{0}^{2}}{(1+k t)^{4}} L\left(J_{1}^{2}(\chi R)-J_{0}(\chi R) J_{2}(\chi R)+J_{0}^{2}(\chi R)\right)$,

where $\chi \equiv 1 /\left(r_{0}(1+k t)\right)$.

According the their physical meanings, $\Phi_{z}$ is the magnetic flux across the surface perpendicular to the axis of a $\mathrm{MC}$, while $\Phi_{\varphi}$ is the magnetic flux across the surface defined by the magnetic axis and the radial direction. Moreover, $R$ denotes the radius of the MC and $L$ is the longitudinal length of the cylindrical structure. By inserting into Eqs. (34a)-(34c) the corresponding expressions for $R$ and $L$, we find

$$
\begin{gathered}
\Phi_{z}=2 \pi R_{0} r_{0} B_{0} J_{1}\left(\frac{R_{0}}{r_{0}}\right)=\text { const., } \\
\Phi_{\varphi}=B_{0} r_{0} L_{0}\left[1-J_{0}\left(\frac{R_{0}}{r_{0}}\right)\right]=\text { const., }
\end{gathered}
$$

and

$H=2 \pi r_{0} R_{0}^{2} B_{0}^{2} L_{0}\left[J_{1}^{2}\left(\frac{R_{0}}{r_{0}}\right)-J_{0}\left(\frac{R_{0}}{r_{0}}\right) J_{2}\left(\frac{R_{0}}{r_{0}}\right)+J_{0}^{2}\left(\frac{R_{0}}{r_{0}}\right)\right]=$ const.

From these results, it follows that the obtained solutions ensure the conservation of magnetic flux and helicity inside the cylindrical MC described by our model. 


\section{Radially expanding MCs}

We now attempt to find solutions for the physical variables when only the radial size of the MC increases. One can see that the solutions in this case do not remain self-similar, although initially a self-similar expansion is assumed in the radial direction.

We consider MCs that are expanding only radially i.e., for which $V_{z}=0$. In this case, Eq. (14) implies that $\dot{\Phi}=0$ or $\delta=-1$. The case with $\dot{\Phi}=0$ corresponds to the stationary state, which is trivial. We consider the case when $\dot{\Phi} \neq 0$ but $\delta=-1$. To provide a consistent time-scaling of all terms in Eq. (22), we have to satisfy

$Q_{\varphi} Q_{\varphi}^{\prime}+\frac{Q_{\varphi}^{2}}{\xi}=\frac{Q_{\varphi}}{\xi} \partial_{\xi}\left(\xi Q_{\varphi}\right)=0$.

An analysis of Eq. (6) $(\xi=r / \Phi(t))$, Eq. (7a) $\left(B_{\varphi}=\Phi^{\delta} Q_{\varphi}(\xi)\right)$, and Eq. (36a) leads to the expression

$\frac{1}{r} \partial_{r}\left(r B_{\varphi}\right)=0$,

where, it is taken into account that $\partial_{r}=\partial_{\xi} / \Phi(t)$.

From Eq. (36b), we can conclude that

$B_{\varphi}=\frac{C}{r}$,

where $C=$ const.

We note that the expression for $B_{\varphi}$ is characterized by a singularity along the axis $(r=0)$. It seems reasonable to conclude that, if we do not consider the axial stretching of self-similarly evolving MCs, we cannot obtain a physically valid solution for the $B_{\varphi}-$ component along the axis of the MC.

\section{Numerical study: higher density and higher plasma $\beta$ case}

We investigate the evolution of MCs in a medium by applying the model described in Sect. 2. For this purpose, the Lagrangian numerical MHD code "Graale" (Finn et al. 2004) is used, which enables us to check whether the aforementioned solutions maintain their self-similarity when they propagate in a medium. In the numerical code, we assume azimuthal and cylindrical symmetries and that the magnetic structure expands uniformly in the longitudinal direction, in other words that $V_{z}=z \dot{L} / L$, where $L$ is the length of the cylinder. An analysis of Eqs. (20a)-(20c) and Eq. (19) shows that the derived expression for the longitudinal velocity coincides with the one implemented in the code.

The assumptions of cylindrical and azimuthal symmetries and and the prescription of the particular character of the longitudinal motions infer the one dimensional numerical simulations. In the numerical runs, the units of the physical parameters are chosen to be the unit length $L_{\text {unit }}=0.1 \mathrm{AU}=$ $15 \times 10^{6} \mathrm{~km}$ (which is the order of the MC's radius at $1 \mathrm{AU}$ ), the unit magnetic field $B_{\text {unit }}=3 \mathrm{nT}$, and the unit number density $n_{\text {unit }}=10 \mathrm{~cm}^{-3}$. After taking into account that the proton mass $m_{\mathrm{p}} \approx 1.7 \times 10^{-27} \mathrm{~kg}$, one finds that the unit mass density is $\rho_{\text {unit }}=$ $m_{\mathrm{p}} n_{0}=0.8 \times 10^{-14} \mathrm{~kg} / \mathrm{m}^{3}$. The unit speed is the Alfvén speed corresponding to $\rho_{\text {unit }}$ and $B_{\text {unit }}$ i.e., $V_{\text {unit }}=V_{0 A}=20.5 \mathrm{~km} \mathrm{~s}^{-1}$, which is of the order of the MC's edge expansion velocity in the frame of the MC (Vandas et al., 2009), and the unit time $t_{\text {unit }}=\frac{L_{\text {unit }}}{V_{\text {unit }}}=200 \mathrm{~h}$. A domain with $R_{\min }=0$ and $R_{\max }=10$ is discretised into 2000 grid cells. The time step used in the simulations is $\Delta t=5 \times 10^{-7}$ (where $R_{\min }, R_{\max }$, and $\Delta t$ are given in units introduced above i.e., $L_{\mathrm{unit}}$ and $\left.t_{\mathrm{unit}}\right)$. Open boundary conditions are applied. Inside the calculation domain, we introduce initial conditions for the physical variables in the two different regions of inside and outside the $\mathrm{MC}$.

The solutions inside and outside the magnetic structure evidently should satisfy the following jump conditions across the surface of any MC

$\left[\varrho v_{r}\right]=0$,

$\left[\varrho v_{r}^{2}+p+\frac{1}{8 \pi} B^{2}\right]=0$.

$\left[\frac{1}{2} \varrho v_{r}^{3}+\left(\frac{\gamma p}{\gamma-1}+\frac{1}{4 \pi} B^{2}\right) v_{r}\right]=0$,

and finally

$\left[\varrho v_{r} v_{t}\right]=0$.

Where [-] denotes the jump of the quantity between the brackets across the surface of the $\mathrm{MC}$ and $v_{r}=V_{r}-V_{\mathrm{s}}$, where $V_{r}$ and $V_{\mathrm{s}}$ are the plasma and the MC's surface velocity, respectively, while $v_{\mathrm{t}}$ denotes the plasma velocity tangential to the surface of the MC. Equations (28a) and (26) show that $v_{r}=0$, which is logical for ideal MHD. Equations (37a), (37c), (37d) are satisfied for arbitrary values of the plasma density and Eq. (37b) leads to the condition

$\left[p+\frac{1}{8 \pi} B^{2}\right]=0$.

We know that the plasma mass density inside the MCs is lower than outside them and the plasma $\beta$ within a MC is lower than in the ambient plasma. We therefore consider $\beta \sim 1$ in the ambient environment and $\beta \sim 0.1$ inside the MC. For the magnetic field within the MC, we use the solution expressed by Eqs. (33a)(33c). For the magnetic field outside the MC, we assume that the azimuthal component of the magnetic field $B_{\varphi \text { out }}=0$, while the longitudinal component $B_{z o u t}$ is uniform. We also assume that the mass density and the thermal pressure are uniform in both regions of the computational domain.

Bearing in mind these assumptions and the jump conditions in Eqs. (37)-(38), one can find explicit expressions for the plasma pressure and magnetic field outside the MC.

Figure 1 represents the numerical solutions for the plasma mass density and velocity, while Fig. 2 shows solutions for the magnetic field components at different moments in time. In panels $a$ and $b$, we plotted the dimensionless values of the density, velocity, and magnetic field. On panels $\mathrm{c}$ and $\mathrm{d}$, we present the dependence of the modified values of the physical parameters on the self-similar variable is presented.

The dependence of the modified mass density, velocity, and dependence of the modified magnetic field components on the self-similar variable clearly shows that our solutions maintain their self-similarity in the course of the MC expansion.

\section{Discussion and conclusions}

We have presented a detailed derivation of a class of selfsimilar analytic solutions of the MHD equations for both radially and axially expanding MCs and a numerical investigation of these solutions. The usage of the self-similar approach is quite common for the modeling of various kinds of solar plasma structures, flows, and eruptions (Low 1982; Osherovich 1993; 

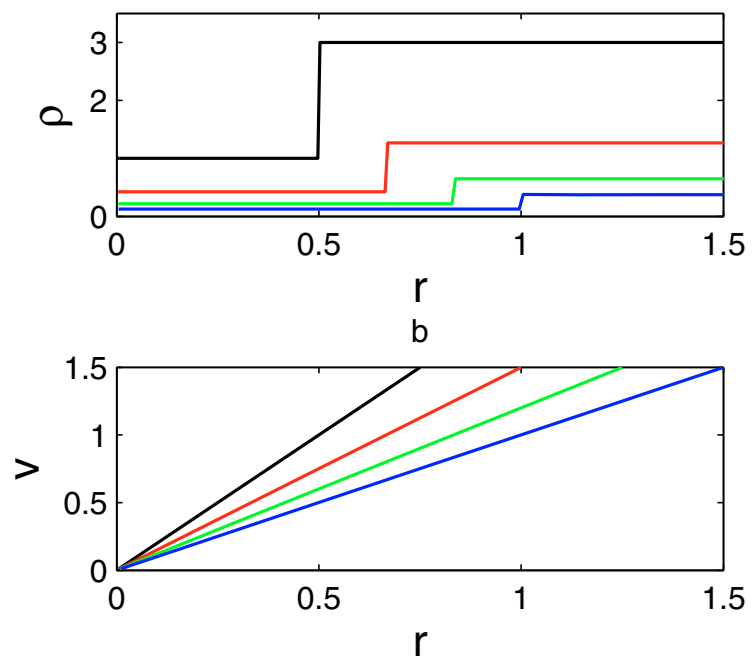

C

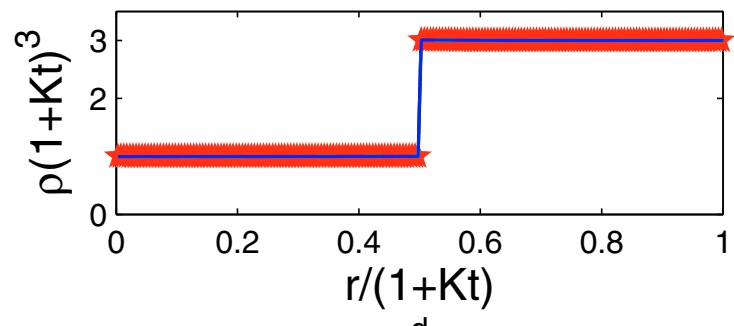

d

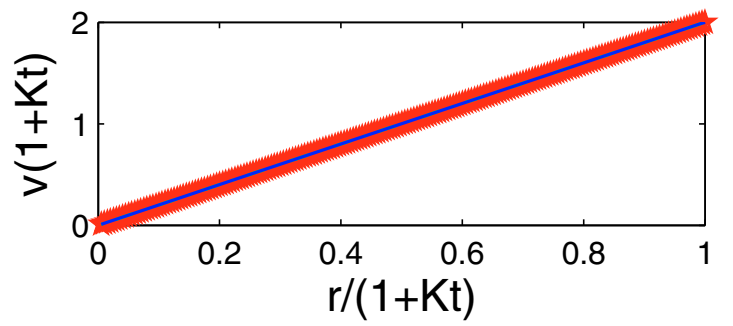

Fig. 1. Snapshots of the evolution of the plasma density and velocity field. Panel a) represents the dependence of the plasma density on the radial coordinate for four moments in time; panel b) shows the dependence on the plasma velocity on the radial coordinate for four time moments; panel c) and panel d) illustrate the dependence of the modified density and the modified velocity, respectively, on the self-similar variable. Parameter values for this case are $k=2, B_{0}=1, \varrho_{\text {out }}=3 \varrho_{\text {in }}, \beta_{\text {in }}=0.1, \beta_{\text {out }}=1$, and $\Phi_{0}=1$. Black line corresponds to $t=0$, red line represents the moment $t=0.5 / 3$, green line shows the time moment $1 / 3$, and blue line corresponds to the moment $t=0.5$.
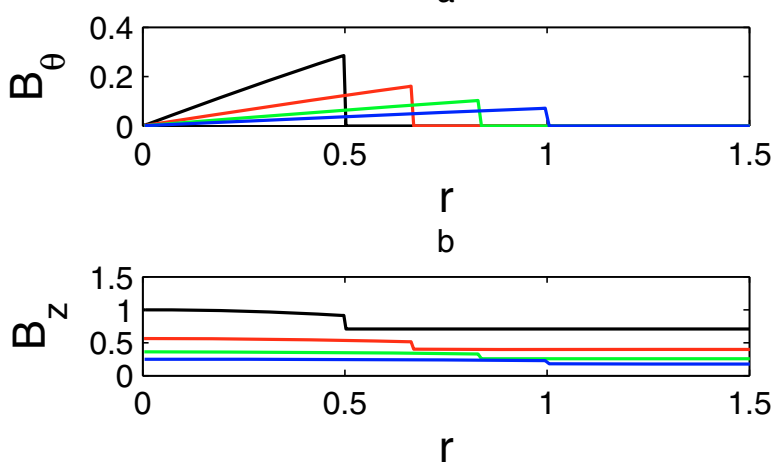

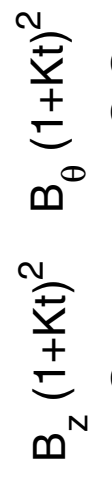

C

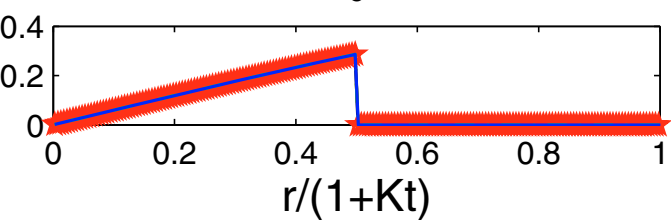

d

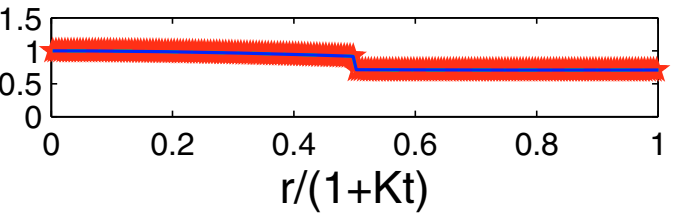

Fig. 2. Snapshots of the evolution of the magnetic field components. Panel a) represents the dependence of the azimuthal component of the magnetic field on the radial coordinate for four time moments; panel b) shows the dependence of the $z$-component of the magnetic field on the radial coordinate for four time moments; panels c) and $\mathbf{d}$ ) illustrate the dependence of the modified azimuthal and $z$-components of the magnetic field, respectively, on the self-similar variable. Parameter values for this case are $k=2, B_{0}=1, \varrho_{\text {out }}=3 \varrho_{\text {in }}, \beta_{\text {in }}=0.1, \beta_{\text {out }}=1, r_{0}=1$, and $\Phi_{0}=1$. Black line corresponds to $t=0$, red line represents the moment $t=0.5 / 3$, the line indicating the time moment $1 / 3$, and blue line corresponds to the moment $t=0.5$.

Farrugia et al. 1995; Nakwacki et al. 2008; Shapakidze et al. 2010). In most previous studies, however, only the radial expansion of the MCs was considered. In the present study, we have taken into account also the axial stretching of the MCs, which is a common observed feature of at least some MCs. We have obtained explicit analytical expressions for the magnetic field, the plasma velocity, the density, and the plasma pressure. Our solutions essentially maintain their self-similar nature during the course of their evolution and propagation through the solar wind. These solutions are complete and well-defined, fully analytic, and, moreover, in the absence of the longitudinal expansion, our solutions self-consistently match the analytic solutions derived by other authors (Farrugia et al. 1995).

For the class of solutions introduced by Eqs. (7a)-(7d), the assumptions of self-similarity and both axial and azimuthal symmetry lead imply that $\Phi(t)$ is a linear function of time (where
$\Phi(t)$ is the time-dependent function of the self-similar variable $\xi=r / \Phi(t))$. In this case, the forces within the MCs are bound to be balanced. We can thus conclude that the case in which the magnetic structures are characterized by a low plasma- $\beta$, corresponds to the force-free magnetic field case. This result also agrees with the conclusion of previous studies. We therefore believe that this is a correct and proper time-dependent generalization of the widely used stationary Lundquist model (Lundquist 1950). Vandas et al. (2006, 2009) compared the generalized Lundquist model with observations and found good agreements between this classic model and the experimental data.

It must be emphasized that our study is not the only one in which the axial stretching of the MCs is taken into account together with their radial expansion. As a matter of fact, Shimazu \& Vandas (2002) also considered MCs with similar properties. In this particular paper, the authors used the mathematical approach 
introduced by Osherovich et al. (1995). To separate the timedependent parts of the solutions from multiplicative functions of the self-similar variable only, Shimazu \& Vandas (2002) adopted a so-called "separable magnetic" field, which was introduced in Osherovich et al. (1995). The approach of Osherovich et al. (1995), was found to be quite restrictive because it requires an ad hoc relation between the different components of the magnetic field. To separate the time-dependent part from the coordinatedependent parts of the momentum equation, in addition to the polytropic law, the authors introduced a specific mathematical expression for the thermal pressure (see Eq. (17), Osherovich et al. 1995). The aforementioned expression relates pressure and mass density (see Eqs. (13)-(17) in Osherovich et al. 1995). In contrast, in our study, we used Low's approach (1982) and required a similar time-scaling for all parts of the Lorentz force and the force caused by the gradient of the thermal pressure. We argue that our approach is more general and places less nonphysical restrictions upon the physical parameters.

Another difference of the results presented here with those of Shimazu \& Vandas (2002) is with the temporal expansion scaling. Shimazu \& Vandas assume that the longitudinal and radial expansions have the same time scaling, while in our work this is not assumed but rather logically follows as the by-product of the accurate solution of the MHD equations. In their paper, the time-dependent function of the self-similar variable is characterized by a linear dependence on time only when the thermal pressure is zero, while we have derived an explicit expression of this time-dependent function of the self-similar variable and demonstrating that $\Phi$ is a linear function of the time variable and does not depend on the character of the pressure function.

Nevertheless another difference between the results presented here and those of Shimazu \& Vandas (2002) is related to the structure of the MC magnetic field. To derive explicit expressions for the magnetic field, Shimazu \& Vandas assumed that the magnetic structure of the MC is described by a force-free magnetic flux rope. In our study, however, we found an explicit expression for the magnetic field. We have derived ordinary differential equations (Eqs. (30a)-(30b)) for the functions describing the components of the magnetic field, after solving the equation of motion for the case corresponding to a low plasma $\beta$ within the MC. For a particular type of parameters, we have found explicit, analytical solutions for the components of the magnetic field (Eqs. (33a)-(33c)). We note that these expressions are a time-dependent generalization of the well-known Lundquist solutions (Lundquist 1950; Burlaga 1988).

Our model implies the conservation of magnetic flux and helicity by design, which is satisfactory and in good agreement with previous investigations (Nakwacki et al. 2008; Demoulin \& Dasso 2009; Kumar \& Rust 1996).

To confirm the validity of our solutions, we investigated the dynamics of magnetic clouds numerically. In the numerical code "Graale", we have introduced our self-similar solutions as initial conditions. The numerical results obtained have shown that during the evolution and propagation of these MCs, their physical variables maintained their self-similar character. This circumstance was illustrated by Figs. 1 and 2 .

The class of solutions found in this paper is obviously quite idealized. The assumptions about the self-similar evolution and the consideration of a cylindrical symmetric structure are quite well-justified, but real MCs show self-similar coherence and cylindrical symmetry only approximately. Hence, in a future study it would be reasonable and interesting to consider more realistic configurations. There are several issues related to the model that should be tested and generalized in any forthcoming study:

1. Our assumptions, as in previous investigations (Low 1982; Farrugia et al. 1995; Finn et al. 2004; Shapakidze et al. 2010), for the systems where entropy is conserved, restrict the value of the polytropic index $\gamma=4 / 3$. We would like to develop a model that helps us to avoid this restriction.

2. Our model describes the plasma dynamics only inside the MC. In the near future, we plan to investigate the interaction of an MC with its environment by constructing consistent solutions of the MHD equations outside the MC.

3. We investigated the obtained analytical solutions numerically using a 1D MHD code and a simple model for the flow outside the MC was implemented. In any future study we also study the MC evolution with 3D numerical codes, where a more complicated and realistic background flow could be implemented (in preparation). In this case the derived solutions could be used as the initial state of the 3D numerical simulation codes. We would like to study the different possible boundary conditions on the surface of the magnetic cloud.

Acknowledgements. These results were obtained in the framework of the projects GOA/2009-009 (K.U. Leuven), G.0304.07 (FWO-Vlaanderen) and C 90347 (ESA Prodex 9). Financial support by the European Commission through the SOLAIRE Network (MTRN-CT-2006-035484), Georgian National Science Foundation grant GNSF/ST06/4-096 and funding from the European Commission's Seventh Framework Programme (FP7/2007-2013) under the grant agreement SOTERIA (project n 218816, www. soteria-space.eu) are gratefully acknowledged. AR acknowledges support of the Abdus Salam ICTP through the Senior Associate Member reward and support of the "Belgian Science Policy" (BELSPO) through a 2009 Fellowship To Non-EU Researchers.

\section{Appendix A:}

We provide some more details of derivation of Eqs. (21) and (22).

During the process of derivation, we found the

$\partial_{r}=\frac{1}{\Phi} \partial_{\xi}$,

$\partial_{t}=-\frac{\xi}{\Phi} \dot{\Phi} \partial_{\xi}$

These relations follow from Eq. (6).

Equation (21) is the radial component of the equation of motion in Eq. (4). In cylindrical coordinates, the left term of Eq. (4) can be written as follows

$\rho\left[\partial_{t}+(\boldsymbol{V} \cdot \nabla) V_{r}\right]=\rho\left[\partial_{t} V_{r}+V_{r} \partial_{r} V_{r}\right]$.

If we take into account Eq. (6) and expression for $V_{r}$ Eq. (11), we obtain the expressions

$$
\begin{aligned}
\partial_{t} V_{r} & =r \frac{\ddot{\Phi}}{\Phi}-r\left(\frac{\dot{\Phi}}{\Phi}\right)^{2}, \\
V_{r} \partial_{r} V_{r} & =r\left(\frac{\dot{\Phi}}{\Phi}\right)^{2} .
\end{aligned}
$$

After substitution of Eqs. (A.4) and (A.5) into Eq. (A.3), we get:

$\rho\left[\partial_{t}+(\boldsymbol{V} \cdot \nabla) V_{r}\right]=\rho \xi \ddot{\Phi}$.

If we combine of Eqs. (7c) and (A.6) we obtain

$\rho\left[\partial_{t}+(\boldsymbol{V} \cdot \nabla) V_{r}\right]=\Phi^{\alpha} \xi \ddot{\Phi} \tilde{\rho}$, 
which is the left term of Eq. (21).

To derive the first part of the right term of Eq. (22), we introduce the notation

$\nabla \times \boldsymbol{B} \equiv \boldsymbol{J}$.

After taking into account the expressions for the magnetic field in Eqs. (7a), (7b) with $\sigma=-2$, we get

$J_{r}=\frac{1}{r} \partial_{\varphi} B_{z}-\partial_{z} B_{\varphi}=0$,

$J_{\varphi}=\partial_{z} B_{r}-\partial_{r} B_{z}=-\partial_{r} B_{z}=-\frac{Q_{z}^{\prime}}{\Phi^{3}}$,

and

$J_{z}=\frac{1}{r} \partial_{r}\left(r B_{\varphi}\right)-\frac{1}{r} \partial_{\varphi} B_{r}=\frac{1}{r} \partial_{r}\left(r B_{\varphi}\right)=\frac{Q_{\varphi} \Phi^{\delta-1}}{\xi}+Q_{\varphi}^{\prime} \Phi^{\delta-1}$.

Since we know expressions for vector $\boldsymbol{J}$, we can derive the vectorial product of $\boldsymbol{J}$ and $\boldsymbol{B}$, which represents the first part of the right term of Eq. (22).

Combining Eq. (A1) with the expression for pressure given in Eq. (7d) leads to the expression of the second part of the right term of Eq. (22).

\section{References}

Berdichevsky, D. B., Lepping R. P., \& Farrugia, C. J. 2003, Phys. Rev. E, 67, 036405

Bothmer, V., \& Schwenn, R. 1998, Ann. Geophys., 16, 1
Burlaga, L. F., Sittler, E., Mariani, F., \& Schwenn, R. 1981, J. Geophys. Res., 86,6673

Burlaga, L. F. 1988, J. Geophys. Res., 93, 7217

Burlaga, L. F. 1991, in Physics of the Inner Heliosphere II: Particles, Waves, and Turbulene, ed. R. Schwenn, \& E. Marsch (New York: Springer), 1

Chandrasekhar, S. 1956, Proc. Nat. Acad. Sci., 42, 1

Demoulin, P. 2008, Ann. Geophys., 26, 3113

Demoulin, P., \& Dasso, S. 2009, A\&A, 498, 551

Farrugia, C. J., Burlaga, L. F., Osherovich, V. A., et al. 1993, J. Geophys. Res., 98, 7621

Farrugia, C. J., Osherovich, V. A., \& Burlaga, L. F. 1995, J. Geophys. Res., 100, 12,293

Finn, J. M., Lapenta, G., \& Li, H. 2004, Phys. Plasmas, 11, 2082

Klein, L. W., \& Burlaga, L. F. 1982, J. Geophys. Res., 87, 613

Kumar, A., \& Rust, D. M. 1996, J. Geophys. Res., 101, 15, 667

Lepping, R. P., Burlaga, L. F., \& Jones, J. A. 1990, J. Geophys. Res., 95, 11 957

Low, B. C. 1982, ApJ, 254, 796

Lundquist, S. 1950, Arkiv för Fysisk, 2, 361

Nakwacki, M. S., Dasso, S., \& Mandrini, C. H. 2008, J. Atmosph. Sol-Ter. Phys., 70,1318

Osherovich, V. A., Farrugia, C. J., \& Burlaga, L. F. 1993, J. Geophys. Res., 98, 13,225

Osherovich, V. A., Farrugia, C. J., \& Burlaga, L. F. 1995, J. Geophys. Res., 100, 12,307

Romashets, E., Vandas, M., \& Poedts, S. 2006, in Proc. ESA SP-617, ed. H. Lacoste, \& L. Ouwehand, 144

Romashets, E., Vandas, M., \& Poedts, S. 2007, A\&A, 466, 357

Shapakidze, D., Debosscher, A., Rogava, A., \& Poedts, S. 2010, ApJ, 712, 565

Shimazu, H., \& Vandas, M. 2002, Earth Plan. Space, 54, 783

Vandas, M., Romashets, E. P., Watari, S., et al. 2006, Adv. Space Res., 38, 441

Vandas, M., Geranios, A., \& Romashets, E. P. 2009, Ap\&SS, 5, 35 